\title{
Clinical impact of repolarization changes in supine versus upright body position
}

\author{
Susanne Markendorf ${ }^{1}$, Thomas F. Lüscher ${ }^{2,3}$, Jin-Hong Gerds-Li ${ }^{4}$, \\ Felix Schönrath ${ }^{5,6}$, Christian Marc Schmied ${ }^{1 *}$ \\ ${ }^{1}$ Division of Cardiology, University Hospital Zurich, Switzerland \\ ${ }^{2}$ Cardiology, Royal Brompton and Harefield Hospitals and Imperial College, London, United Kingdom \\ ${ }^{3}$ Center for Molecular Cardiology, University of Zurich, Switzerland \\ ${ }^{4}$ Division of Cardiology, Deutsches Herzzentrum Berlin, Germany \\ ${ }^{5}$ Department of Cardiothoracic and Vascular Surgery, Deutsches Herzzentrum Berlin, Germany \\ ${ }^{6}$ DZHK (German Center for Cardiovascular Research), Partner Site Berlin, Germany
}

\begin{abstract}
Background: The impact of postural changes on various electrocardiography (ECG) characteristics has only been assessed in a few small studies. This large prospective trial was conducted to confirm or refute preliminary data and add important results with immediate impact on daily clinical practice.

Methods: ECGs in supine and upright position from 1028 patients were analyzed. Evaluation was made according to changes in T-wave vector and direction, ST-segment deviation, heart rate, QT interval and QTC interval was performed. Findings were correlated with the medical history of patients.

Results: Positional change from supine to upright resulted in a significantly increased heart rate $(8.05 \pm 7.71 \mathrm{bpm})$ and a significantly increased QTc interval after Bazetts $(18 \pm 23.45 \mathrm{~ms})$ and Fridericas (8.84 \pm 17.30$)$ formula. In the upright position significantly more T-waves turned negative (14.7\%) than positive (5.7\%). ST elevation was recorded in only $0.4 \%$ and ST depression in not more than $0.2 \%$ of all patients.

Conclusions: The majority of the patients do not show significant morphological changes in their ECG by changing the body position from supine to upright. Changes of QTc time instead, are significant and the interval might be overestimated in upright. Therefore assessment of the QTc interval should strictly be done in a supine position. (Cardiol J 2018; 25, 5: 589-594)
\end{abstract}

Key words: electrocardiography, electrophysiology, from supine to upright, long-QT, QTc variations

\section{Introduction}

Currently, 12-lead resting electrocardiogram $(\mathrm{ECG})$ is the standard modality to investigate the electrophysiology of humans. A standard ECG is routinely recorded in the supine position [1]. The electrocardiac polarization is detected from the body surface and it is an established fact that positional changes of the heart with a constant position of the ECG leads frequently leads to alterations of various ECG findings assessed in the supine position [2]. These situations may lead to clinical misinterpretation, with potentially fatal consequences. In multiple scenarios in daily clinical practice an ECG recording in supine position is not possible. Some patients may not be able to lie down (e.g. patients with orthopnoea or orthopaedic disease) while classical exercise testing (either on

Address for correspondence: Dr. Susanne Markendorf, University Heart Center - University Hospital Zurich, Raemistrasse 100, CH-8091 Zürich, Switzerland, tel: 0041'44'934'2364, fax: 0041'44'255'1616, e-mail: s.markendorf@icloud.com

Received: 28.06.2017 Accepted: 01.11.2017

*Both authors contributed equally. 
a bicycle or treadmill) is routinely performed in the upright position. Therefore, it seems surprising that only a few studies have assessed the impact of body position regarding various recent ECG parameters.

Swenson et al. [3] created a geometric model consisting of triangular elements representing the heart and torso tank surfaces and supposed significant potential variations, especially ST-segment voltage changes by postural change. These variations seemed to be more pronounced for a heart exhibiting increased ischemic potential. It was hypothesized that this potential could even be large enough to mask or mimic signs of ischemia in the ECG. Furthermore, few prospective studies, with only 12,40 and 54 subjects, respectively [2, 4, 5], investigated the effect of postural change in body position on QT and QTc interval. However, all these data were gained either from geometric models or only small cohorts. Thus, these results must be interpreted as somewhat clinically preliminary.

\section{Methods}

\section{Study population}

One thousand and twenty eight patients were prospectively examined at the Cardiology Outpatient Clinic of the University Heart Centre Zurich, Switzerland. After patients had given informed consent, ECGs (see details below) were performed. All patients had different cardiologic and non-cardiologic diagnoses that would have made it necessary to record an ECG on that specific day. Exclusion criteria were age $<18$ years and/or the incapability of sitting unassisted in an upright position. All patients were hemodynamically stable without chest pain at the time of recording. The trial was approved by local ethics authorities (KEK Ref-No: 2012-0522)

\section{Subgroups}

For the purpose of data analysis the study population was further divided into the following three subgroups: patients with complete bundle branch block (left or right, QRS interval $\geq 120 \mathrm{~ms}$ ), patients with incomplete bundle branch block and patients without any of these pathologies. Fourteen patients with atrial fibrillation were excluded in this subgroup analysis.

Given the fact that T-wave inversions in leads III, aVR and V1 may be physiologic [6], focus was particularly on postural ECG changes in a subgroup where ECGs with T-wave changes in one or several of these three leads were excluded, it was thus called the subgroup of T-wave changes in "relevant leads".

Finally, the positional changes were to be correlated with the clinical background of the patients. For the purpose of this study focus was on the specific group of patients with coronary heart disease (CHD) and assessment of whether these patients showed particular ECG changes compared to patients with no known CHD.

\section{ECG execution}

All 12-lead ECGs were performed by two experienced and particularly skilled nurses at the Cardiology Outpatient Division of the University Heart Centre Zurich. ECGs were recorded on the Schiller Cardiograph model AT-10 Plus (Schiller AG, Baar/Switzerland), programmed for a paper speed of $25 \mathrm{~mm} / \mathrm{s}$. In this cardiograph QT time is calculated based on the average beat [7]. Authoritative for the algorithmic implementing is the first QRS onset and the last $T$ wave activity of a $10 \mathrm{~s}$ record over 12 standard leads. All normal beats, excluding extra systole are extracted and an average beat is generated. Out of that average beat QT time is measured. All of these automatically generated beats were manually remeasured. For the majority, no differences could be seen. In cases of a difference between the manually and the automatically measured QT length, only manual ones were considered for the study data. The QTc interval was calculated using Bazett's formula [8] QTc = $=\frac{Q T}{\sqrt{R R}}$ and Fridericas [8] formula QTc $=\frac{Q T}{\sqrt[3]{R R}}$. Immediately after a 12-lead standard ECG had been recorded in the supine position, the patient was asked to sit up and the second ECG was recorded after 2 min without the position of the electrodes being changed.

\section{Data documentation}

All ECGs were analyzed descriptively and blinded (regarding posture) twice, once by the same senior consultant cardiologist and once by the same ECG experienced master's student. Afterwards the two analyses were compared and all discrepancies were discussed to consider the best solution for a final consensus. Changes of T-wave direction and ST segment deviation of at least $1 \mathrm{~mm}$ (positive or negative) in at least one lead between supine and upright were documented. However, most ECGs had multiple changes in different leads, which were all documented.

Furthermore, changes of the heart rate (HR), QT interval and QTc interval after the standard 
Table 1. Cardiac diagnoses of the whole study population.

\begin{tabular}{lc}
\hline Coronary heart disease & $49(4.8 \%)$ \\
Dilatative cardiomyopathy & $4(0.4 \%)$ \\
Non-compaction cardiomyopathy & $1(0.1 \%)$ \\
Hypertrophic cardiomyopathy & $4(0.4 \%)$ \\
Aortic isthmus stenosis & $1(0.1 \%)$ \\
Aortic stenosis & $2(0.2 \%)$ \\
Endocarditis & $4(0.4 \%)$ \\
Myocarditis & $1(0.1 \%)$ \\
Atrioventricular block & $2(0.2 \%)$ \\
AV-NRT & $2(0.2 \%)$ \\
Fabry disease & $2(0.2 \%)$ \\
Tetralogy of Fallot & $1(0.1 \%)$ \\
Heart transplantation & $1(0.1 \%)$ \\
Mitral valve reconstruction & $1(0.1 \%)$ \\
Long QT syndrome & $2(0.2 \%)$ \\
Systemic sclerosis & $4(0.4 \%)$ \\
Takotsubo cardiomyopathy & $1(0.1 \%)$ \\
Takayasu arteritis & $1(0.1 \%)$ \\
Atrial fibrillation (paroxysmal or permanent) & $23(2.3 \%)$ \\
Other supraventricular tachycardia & $1(0.1 \%)$ \\
\hline
\end{tabular}

Data are shown as number (percentage); AV-NRT — atrioventricular nodal reentry tachycardia

clinical correction with Bazett [8] and Fridericas [9] formulas were analyzed.

\section{Statistical analysis}

Quantitative variables are expressed as mean \pm standard deviation and categorical variables as frequencies or percentages. Differences in heart rate, QT interval and QTc interval were evaluated using the Student t-test. Chi-square test was applied to show the significance of cross table analyses. Sign test was used to show the significance of descriptive analyses. The level of statistical significance was defined as a p-value less than 0.05. Analysis was performed using IBM SPSS Statistics (Version 21. Armonk, NY).

\section{Results}

\section{Patient baseline characteristics}

Overall, 1028 ECGs were evaluated: 439 in females with an average age of $55.62 \pm 16.67$ years and 589 in males with an average age of $57.92 \pm$ \pm 15.98 years.

Only 107 (10.4\%) patients of our study population had a background of cardiac disease (Table 1). As already stated, the patients were further divided into the subgroups regarding their initial ECG tracing in supine body position: patients without any of the following conduction anomalies $(n=868)$, patients with complete bundle branch block $(n=45)$ and patients with incomplete bundle branch block $(\mathrm{n}=81)$. Twenty patients with ventricular pacemaker rhythm and 14 patients with atrial fibrillation were excluded for these evaluations.

In another sub-analysis, a subgroup of patients with T-wave changes in clinically "relevant leads" $(\mathrm{n}=86)$ and one of the patients with $\mathrm{CHD}(\mathrm{n}=46)$ were formed. All subgroups were comparable in terms of the evaluated baseline parameters.

\section{ECG characteristics}

Postural change from supine to upright resulted in a significantly increased HR $(8.05 \pm 8 \mathrm{bpm})$. This result was consistent in all subgroups. The QT interval significantly shortened in all subgroups $(-6.07 \pm$ $\pm 17 \mathrm{~ms}$ ), however, the corrected QTc intervals after Bazetts formula (18 $\pm 23 \mathrm{~ms})$ as well as after Fridericas formula $(8.84 \pm 17)$ increased significantly in all subgroups. Table 2 shows detailed QT, QTc interval and HR changes in supine vs. upright position.

No changes of the T-wave vector/direction (from supine to upright position) could be shown in $731(71.1 \%)$ patients.

Leads with $\mathrm{T}$-waves turning negative in an upright position were seen in 151 (14.7\%), T-waves turning positive were seen in 56 (5.4\%) patients. Both phenomena together in one ECG recording were seen in $90(8.8 \%)$ patients. Thus, T-wave inversions after moving to an upright position were observed significantly more often than negative T-waves turning positive $(\mathrm{p}<0.001)$. This finding was equal in the subgroup with partial bundle branch block. When leads III, avR and V1 were excluded, only 86 patients showed positional T-wave changes. After this adaption $66(76.7 \%)$ positive T-waves turned negative and $17(19.8 \%)$ negative T-waves turned positive in the upright position.

Table 3 shows differentiation of the T-wave changes in the various leads.

In the subgroup of 49 patients with CHD 5 (10.2\%) patients showed postural T-wave inversions, while only $2(4.1 \%)$ demonstrated T-waves turning positive. Six patients showed both phenomena in one ECG recording. Thus, a specific pattern in this subgroup could not be shown. Figures 1 and 2 show a supine and an upright ECG from the same patient as an example for a clear T-wave change from positive to negative. This patient was 41 years old, had a one vessel CHD in the left anterior descending and a normal body mass index 
Table 2. Results: QT, QTc and heart rate supine versus upright.

\begin{tabular}{|c|c|c|c|c|}
\hline & Supine & Upright & Average change \pm SD & $\mathbf{P}$ \\
\hline \multicolumn{5}{|c|}{ Overall patients $(n=1028)$} \\
\hline Heart rate $[\mathrm{bpm}]$ & $73.92 \pm 15$ & $82.52 \pm 16$ & $8.05 \pm 8$ & $<0.001$ \\
\hline QT [ms] & $386.74 \pm 39$ & $380.67 \pm 37$ & $-6.07 \pm 17$ & $<0.001$ \\
\hline QTc Bazett [ms] & $426 \pm 31$ & $444 \pm 31$ & $18.00 \pm 23$ & $<0.001$ \\
\hline QTc Friderica [ms] & $412.20 \pm 29$ & $421.36 \pm 28$ & $8.84 \pm 17$ & $<0.001$ \\
\hline \multicolumn{5}{|c|}{ Bundle branch block $(n=45)$} \\
\hline Heart rate $[\mathrm{bpm}]$ & $76.84 \pm 14$ & $82.02 \pm 14$ & $5.18 \pm 6$ & $<0.001$ \\
\hline QT [ms] & $415.78 \pm 42$ & $409.46 \pm 38$ & $-6.31 \pm 13$ & 0.003 \\
\hline QTc Bazett [ms] & $467.31 \pm 29$ & $476.31 \pm 26$ & $9.67 \pm 16$ & $<0.001$ \\
\hline QTc Friderica [ms] & $449.05 \pm 29$ & $452.97 \pm 26$ & $3.93 \pm 12$ & 0.038 \\
\hline \multicolumn{5}{|c|}{ Incomplete bundle branch block $(n=81)$} \\
\hline Heart rate $[\mathrm{bpm}]$ & $78.16 \pm 18$ & $86.36 \pm 18$ & $7.93 \pm 6$ & $<0.001$ \\
\hline QT [ms] & $391.70 \pm 79$ & $376.59 \pm 54$ & $-15.09 \pm 83$ & $<0.001$ \\
\hline QTc Bazett [ms] & $435.11 \pm 30$ & $454.83 \pm 33$ & $19.71 \pm 24$ & $<0.001$ \\
\hline QTc Friderica [ms] & $418.86 \pm 34$ & $425.46 \pm 34$ & $6.60 \pm 37$ & $<0.001$ \\
\hline \multicolumn{5}{|c|}{ Without arrhythmia or conduction disorder $(n=868)$} \\
\hline Heart rate $[\mathrm{bpm}]$ & $73.20 \pm 15$ & $82.18 \pm 15$ & $8.98 \pm 7$ & $<0.001$ \\
\hline QT [ms] & $384.23 \pm 36$ & $378.19 \pm 35$ & $-6.04 \pm 16$ & $<0.001$ \\
\hline QTc Bazett [ms] & $421.30 \pm 27$ & $440.37 \pm 28$ & $19.07 \pm 23$ & $<0.001$ \\
\hline OTc Friderica [ms] & $408.12 \pm 24$ & $418.18 \pm 26$ & $10.06 \pm 18$ & $<0.001$ \\
\hline
\end{tabular}

Data are shown as mean \pm standard deviation (SD).

Table 3. T-wave change in various leads.

\begin{tabular}{|c|c|c|c|c|c|c|c|c|c|c|c|c|}
\hline & V1 & V2 & V3 & V4 & V5 & V6 & I & II & III & aVR & aVL & aVF \\
\hline T-wave turning negative (N) & - & 16 & 19 & 17 & 14 & 10 & 1 & 5 & - & - & 7 & 25 \\
\hline T-wave turning positive (N) & - & 4 & 2 & 0 & 2 & 1 & 1 & 1 & - & - & 12 & 0 \\
\hline
\end{tabular}

Data are shown as number.

of 26.9. Cardiovascular risk factors were smoking, dyslipidemia and positive family history.

The ST segments proved to be unchanged in $1019(99.1 \%)$ patients, while $6(0.6 \%)$ ECGs showed an ST segment elevation and only $2(0.2 \%)$ patients showed a depression of the ST segment in an upright position. One $(0.1 \%)$ patient showed ST segment elevation as well as depression in different leads of the ECG. However, 4 patients with ST segment elevation had a complete bundle branch block, while the 2 patients with ST segment depression showed no specific ECG findings in the baseline ECG recorded in supine position.

\section{Discussion}

Reported herein are different ECG variables in, thus far, the largest community based cohort according to possible postural changes. Additionally, in comparison to previous, smaller studies $[2,4,5]$, additional parameters were added that have not, as yet, been assessed in this context. These variables are of crucial impact for the accurate assessment and treatment of a patient in cases of an emergency or in long-term follow-up.

\section{Heart rate, QT and QTc changes}

One of the main findings of this study was a prolongation of the corrected QT interval in an upright position although HR increased significantly. It is well known that a higher HR leads to a shortening of RR interval and therewith as well to a QT shortening. But in contrast to trials with much smaller study populations [9-11] which postulated stable QTc times by postural change, an increase was also shown of in QTc time in the Bazett and 


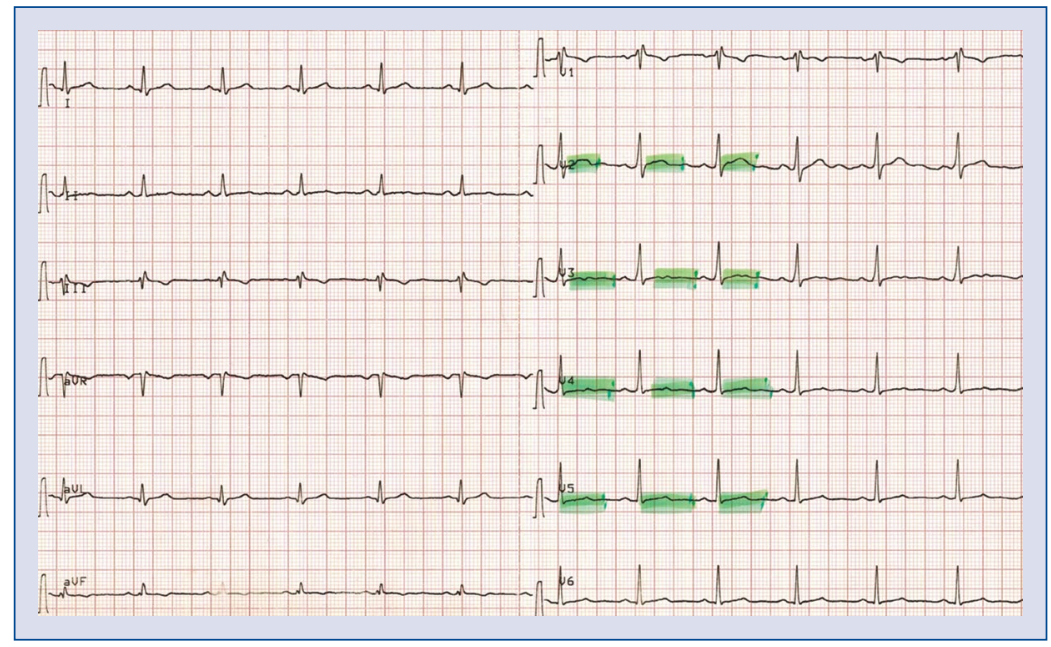

Figure 1. Supine electrocardiogram of 1 patient.

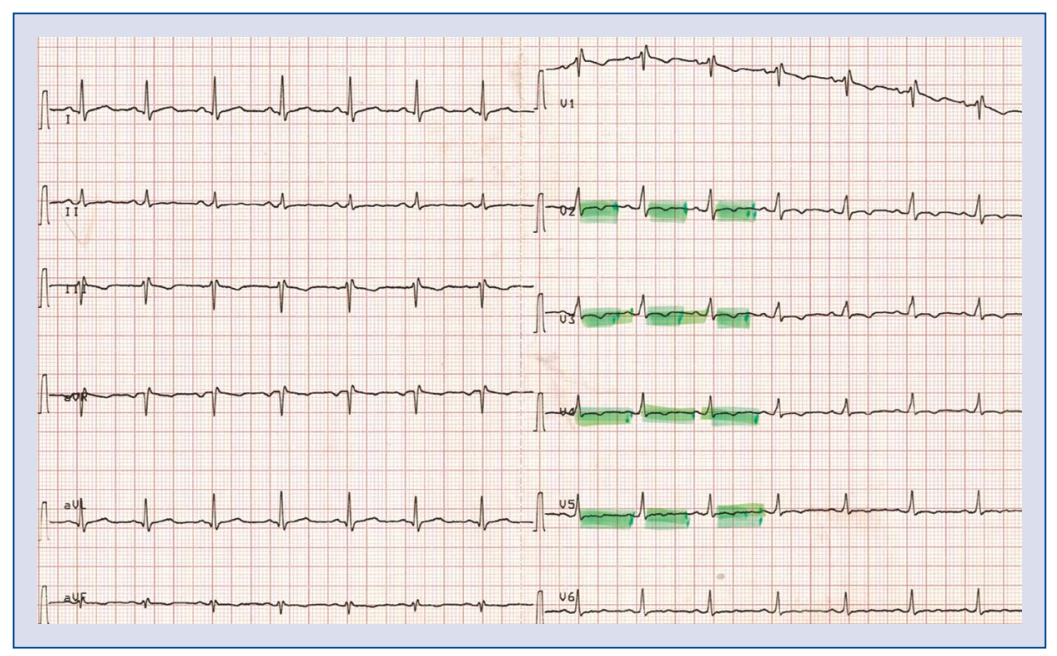

Figure 2. Electrocardiogram in upright body position of the same patient as well as the supine electrocardiogram in Figure 1. T-wave inversions in leads V2-V5 are clearly visible.

Friderica correction formula. It is known that a calculation of QTc interval using Bazett's formula may overcorrect QT interval at fast HRs [9, 12], whereby Friderica's formula seems to have the best correction rate and is more accurate than Bazett's formula in fast as well as in slow HRs [10, 13]. In a trial which investigated the postural change from supine to standing position HR increased by $21.4 \pm$ $\pm 8 \mathrm{bpm}$, while QTc-interval after Bazett increased by $9.6 \pm 9 \mathrm{~ms}$ [5]. In the present trial HR increase was only moderate, interestingly the prolongation of the QTc interval was even more impressive. This effect was reproducable in all subgroups

\section{T-wave and ST segment changes}

The T-wave equaling the repolarization of electrical excitation is of crucial relevance for the accurate interpretation of an ECG. $30 \%$ of our patients demonstrated a change of the $\mathrm{T}$ wave axis in an upright position. This high percentage, suggests that an interpretation of particularly negative T-waves highly depends on the position of the upper body. This aspect underlines the importance of a correct and comparable position of a patient for interpretation of repolarization changes to avoid misinterpretation. Inverted $\mathrm{T}$ waves (in all leads, excluding III, aVR and V1) are highly sug- 
gestive for structural or ischemic heart disease [6]. In more than $22 \%$ of patients, T-wave axis turned negative after sitting up, what therewith mimicked a potential structural heart disease. On the other hand, inverted T-waves turning positive in an upright position, which was the finding in more than $14 \%$ of studied patients, may mask underlying T-wave inversion and thus underlying structural heart disease. However, focusing on a sub-group analysis of 49 patients with known CHD a particular correlation between postural T-wave changes and this specific cardiovascular disease could not be demonstrated.

Deviations of the ST segment are also of high clinical and diagnostic impact, particularly ST segment depressions which may suggest structural or ischemic heart disease, while the consequences of so called "early repolarization" of the ST segment are still a matter of debate [14]. However, in contrast to T-wave changes it has to be emphasized that with less than $1 \%$, postural changes of the ST segments in the present cohort were very rare. This is surprising, as the geometric model of Swenson et al. [3] which suggested more ST segment changes in the present large cohort. Furthermore, based on this model these variations of voltage changes seem to be more pronounced for a heart exhibiting increased ischemic potentials, which could also not be reproduced in this study.

\section{Limitations of our study}

Besides having collected data prospectively from, to date, the largest cohort for the evaluation of postural changes, it has to be recognized, that data collected was only in one center. Furthermore, even with this large cohort, statistical evaluation of interesting subgroups e.g. atrial fibrillation were not possible because of the small number of patients. Since it was chosen to use a cross sectional design for evaluation of the present cohort, a long term follow up of clinical events compared to the documented ECG changes would be a valuable next step to integrate this data in a clinical context.

\section{Conclusions}

The majority of patients did not show significant morphological changes in their ECG by changing the body position from supine to upright. Changes of QTc time instead, are significant and intervals might be overestimated in the upright position. Therefore assessment of the QTc interval should strictly be done in a supine position.
T-wave changes are more frequent than changes of the ST segment. Furthermore, more T-waves turn negative in an upright position, mimicking structural or ischemic myocardial disease, while on the other hand in more than $14 \%$ of cases inverted T-waves turned positive and may therefore mask relevant diseases.

\section{Acknowledgements}

The authors would like to thank Anne Gale for editing of this manuscript.

\section{Conflict of interest: None declared}

\section{References}

1. Madias JE. Comparability of the standing and supine standard electrocardiograms and standing sitting and supine stress electrocardiograms. J Electrocardiol. 2006; 39(2): 142-149, doi: 10.1016/j. jelectrocard.2005.07.006, indexed in Pubmed: 16580409.

2. Williams GC, Dunnington KM, Hu MY, et al. The impact of posture on cardiac repolarization: more than heart rate? J Cardiovasc Electrophysiol. 2006; 17(4): 352-358, doi: 10.1111/j.15408167.2005.00415.x, indexed in Pubmed: 16643354.

3. Swenson DJ, Geneser SE, Stinstra JG, et al. Cardiac position sensitivity study in the electrocardiographic forward problem using stochastic collocation and boundary element methods. Ann Biomed Eng. 2011; 39(12): 2900-2910, doi: 10.1007/s10439-0110391-5, indexed in Pubmed: 21909818.

4. Adams M, Drew B. Body position effects on the ECG. J Electrocardiol. 1997; 30(4): 285-291, doi: 10.1016/s00220736(97)80040-4.

5. Huang MH, Ebey J, Wolf S. Heart rate-Qt interval relationship during postural change and exercise. A possible connection to cardiac contractility. Integr Physiol Behav Sci. 1991; 26(1): 5-17, indexed in Pubmed: 2054298.

6. Drezner J, Ackerman M, Anderson J, et al. Electrocardiographic interpretation in athletes: the 'Seattle Criteria': Table 1. Br J Sports Med. 2013; 47(3): 122-124, doi: 10.1136/ bjsports-2012-092067.

7. Christov II, Simova II. fully automated method for QT interval measurement in ECG. Computers in Cardiology. 2006; 33: 321-324.

8. Bazett HC. An analysis of the time-relations of electrocardiograms. Heart. 1920; 7: 353-370.

9. Fridericia LS. The duration of systole in an electrocardiogram in normal humans and in patients with heart disease. 1920. Ann Noninvasive Electrocardiol. 2003; 8(4): 343-351, indexed in Pubmed: 14516292.

10. Obata Y, Ruzankin P, Ong QiJ, et al. The impact of posture on the cardiac depolarization and repolarization phases of the QT interval in healthy subjects. J Electrocardiol. 2017; 50(5): 640_645, doi: 10.1016/j. jelectrocard.2017.03.001, indexed in Pubmed: 28330682.

11. Sagie A, Larson MG, Goldberg RJ, et al. An improved method for adjusting the QT interval for heart rate (the Framingham Heart Study). Am J Cardiol. 1992; 70(7): 797-801, indexed in Pubmed: 1519533.

12. Vandenberk B, Vandael E, Robyns T, et al. Which QT correction formulae to use for QT monitoring? J Am Heart Assoc. 2016; 5(6), doi: 10.1161/JAHA.116.003264, indexed in Pubmed: 27317349.

13. Davey P. Influence of posture and handgrip on the QT interval in left ventricular hypertrophy and in chronic heart failure. Clin Sci (Lond). 1999; 96(4): 403-407, indexed in Pubmed: 10087248.

14. Macfarlane PW, Antzelevitch C, Haissaguerre M, et al. The Early Repolarization Pattern: A Consensus Paper. J Am Coll Cardiol. 2015; 66(4): 470-477, doi: 10.1016/j.jacc.2015.05.033, indexed in Pubmed: 26205599. 